\title{
Propagation Characteristics of Intra-body Communications for Body Area Networks
}

\author{
Jordi Agud Ruiz, Jiang Xu, Shigeru Shimamoto \\ Graduate School of Global Information and Telecommunication Studies, Waseda University \\ 29-7 building, 1-3-10 Nishi-Waseda, Shinjuku-ku, Tokyo 169-0051 Japan \\ E-mail: jordi.agud@toki.waseda.jp,justinxu@suou.waseda.jp,shima@waseda.jp
}

\begin{abstract}
Body area networks (BAN) will become increasingly important in the future personal communication systems. We believe that intra-body communications (IBC) based on near field electromagnetic waves is a suitable solution for BAN. This paper presents experimental measurements of the frequency and time domain responses in order to investigate the transmission characteristics of the human body as a conductor of low power high frequency radio signals (up to $3 \mathrm{GHz}$ ). In the measurements, several distances between transmitter and receiver (up to a long distance from the head to the foot) and six test people (to study the possible variation results due to physiological body effects) have been considered. Besides analyzing the intra-body channel response, we also aim to define a suitable frequency range for IBC. Finally, this paper also presents an initial evaluation study of several digital modulation schemes in order to investigate the optimal technique for IBC.
\end{abstract}

Keywords- body area networks, intra-body communications, body transmission characteristics, body channel, blind people guidance application

\section{INTRODUCTION}

IBC is a short-range communication between devices in which the human body is used as the signal transmission medium. We believe that IBC will allow the interaction between devices that move with the user body, and between users and the surrounding environment [1]. There are two main solutions for IBC: electric field type [2]-[4] and near field electromagnetic type [5]-[6]. By means of the former solution, data is sent across devices placed on or near the body by using a near field electric field. In the latter solution, the human body is treated as a waveguide with the radio frequency signals propagating through the human body.

The number of potential applications by means of IBC is enormous. We have envisioned applications for handicap people. For example, IBC could be used by blind people as a guidance system when walking in the streets, shopping in department stores or moving inside train stations. In order to implement this service, the blind person should wear an IBC enabled sensor located at the bottom of the shoe. Moreover, an IBC enabling line of sensors should be attached to the surface of the sidewalk. As the Fig. 1 shows, whenever the blind person's shoes step the IBC enabled ground line, communication could be carried out between the IBC enabled foot sensor and the IBC enabled sidewalk. The data received in the foot sensor could be forwarded through the body to an IBC enabled earphone that the blind person could carry, so that he/she could hear the information by voice. The IBC enabled ground line could send information such as the current location (street name, surrounding buildings and establishment, etc.), the destination and time departure of the closest track to his/her location in the train station or the special offers of the section of the supermarket where he/she is at each moment.

Our research is based on the near-field radio solution. In [1], we presented our IBC system model and some initial experiment results whereby we investigated the transmission characteristics of the human body as a conductor of radio frequency signals up to $1400 \mathrm{MHz}$, which it was much higher than the maximum $100 \mathrm{MHz}$ frequency carrier considered in [6]. In the present paper, firstly we investigate the body propagation characteristics considering higher frequency signals (up to $3 \mathrm{GHz}$ ) and bigger distance between transmitter (TX) and receiver (RX) (i.e. TX in the foot and RX in the ear, simulating the guidance application for blind people). Moreover, six test people with different ages and body structures are used in the experiments. On the other hand, this paper also presents experimental results of the performance evaluation of three digital modulation schemes. References [2], [3] and [5] employed mainly OOK, ASK and FSK as modulation schemes because of the easy implementation in the transceiver prototypes. However, there is not any specific study related to the optimal modulation scheme for IBC. For this reason, we aim to evaluate several modulation schemes in order to determine the most suitable one for IBC. We believe

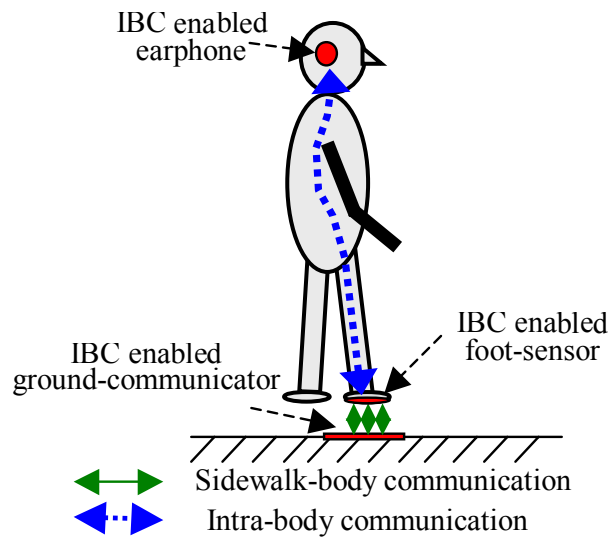

Figure 1. Guidance IBC application scenario for blind people. 
that an optimal modulation scheme for IBC would allow an increase of the low bit rate achieved in the current related works (up to $9.6 \mathrm{Kbps}$ in [5]).

The paper is organized as follows. Section II describes the experiment setup for the investigation of the body transmission characteristics, and in Section III, the experiment results are presented. Sections IV and V show respectively the experiment setup and the results of the evaluation of several modulation schemes applied to intra-body channels. Finally, Section VI contains the conclusions of this paper.

\section{EXPERIMENT SETUP For THE INVESTIGATION OF THE BODY PROPAGATION CHARACTERISTICS}

The experiments were performed in a classroom with only desks and chairs. The frequency and time domain responses were measured using a network analyzer. Fig. 2 shows the measurement system scenario where the radio signals were generated in the network analyzer, input in the body through the TX, received in the $\mathrm{RX}$ and evaluated in the network analyzer. Table I shows the main measurement setup parameters. The input signal power was selected based on the study about the possible health effects of exposure to electromagnetic fields carried out in [7]. This study recommended a basic limit exposure of $0.08 \mathrm{~W} / \mathrm{kg}$ for the human body. Considering an average weight of $65 \mathrm{~kg}$, the maximum transmit signal power could be $37 \mathrm{dBm}$. Even so, we used a lower input power of just $15 \mathrm{dBm}$. Six test people (three males and three females) with different ages (from 24 to 32 years old) and different body structures $(1.50 \mathrm{~m}$ to $1.80 \mathrm{~m}$ height range and $45 \mathrm{~kg}$ to $80 \mathrm{~kg}$ weight range) were used in the experiments. TX and RX were composed by a signal electrode and a ground plane (GND).

The signal electrode was implemented with a round copper plate that touched the skin. Copper was selected as the electrode material because of the good conductivity and also due to the study of contact impedance between body and electrodes using seven commercial metals (aluminum, copper,

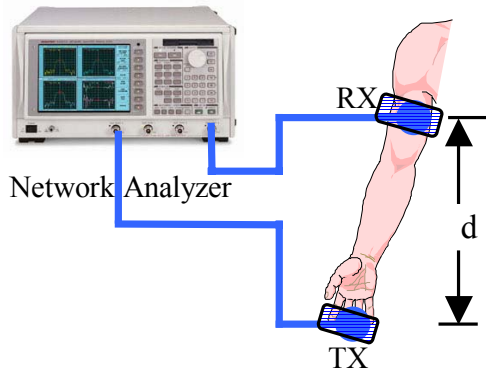

Figure 2. Measurement system scenario.

TABLE I. MAIN MEASUREMENT SETUP PARAMETERS

\begin{tabular}{l|l}
\hline Parameter & Value \\
\hline Frequency range $[\mathrm{MHz}]$ & $1 \sim 3000$ \\
\hline Frequency points & 1201 \\
\hline Transmit power $[\mathrm{dBm}]$ & 15 \\
\hline Distance between TX and RX $[\mathrm{cm}]$ & $20 \sim 155$ \\
\hline Test persons & 6 \\
\hline
\end{tabular}

bronze, brass, stainless steel, nickel silver and $\mathrm{Ag}-\mathrm{AgCl}$ ) carried out in [5] that showed that the contact impedance of the electrodes was independent of the kind of metal. We investigated the optimal TX and RX configuration by considering TX and/or RX with or without GND electrode touching the skin and changing the signal and GND electrode diameter sizes (from $1 \mathrm{~cm}$ to $3 \mathrm{~cm}$ ), the size of the GND circuit board $(10 \mathrm{~cm}$ by $5 \mathrm{~cm}, 5 \mathrm{~cm}$ by $5 \mathrm{~cm}$ and $2 \mathrm{~cm}$ by $2 \mathrm{~cm})$, and the distance between the signal electrode and the circuit board (from $0.7 \mathrm{~cm}$ to $2.1 \mathrm{~cm}$ ). Based on [1] and some subsequent investigations, we concluded that the best TX and RX configuration, in terms of the lowest path loss, was composed of only signal electrode touching the skin of $2 \mathrm{~cm}$ diameter and a $10 \mathrm{~cm}$ by $5 \mathrm{~cm}$ GND circuit board distant $1.4 \mathrm{~cm}$ from the signal electrode.

We defined two different measurement models that are shown in Fig. 3: sitting and standing model. In the sitting model, the test person sat on the chair, the TX position was always fixed and mounted on the table, and the RX was located along the arm. The standing model was defined based on the IBC application scenario for blind people. The test person's foot stood on the TX and several RX measurement positions were settled (e.g. waist and ear).

\section{EXPERIMENT RESULTS AND DISCUSSIONS}

To investigate the radio propagation characteristics of the human body, we analyze the frequency and time domain responses obtained for both measurement models. Moreover, we compare these results with the measurements obtained in case of air propagation (i.e. when TX and RX don't touch the body).

\section{A. Frequency Domain Response}

Figs. 4 and 5 show the results of the sitting model (when the distance between TX and RX is $20 \mathrm{~cm}$ and $55 \mathrm{~cm}$ ) and the standing model (waist-foot and ear-foot distances between TX and $\mathrm{RX}$ ) respectively. The horizontal axis represents the frequency in $\mathrm{MHz}$ (from $1 \mathrm{MHz}$ to $3 \mathrm{GHz}$ ) and the vertical axis represents the received signal power in $\mathrm{dB}$. Each figure is composed by the air propagation response and the average intra-body propagation response of the six test people. Both figures show that the attenuation of the received signal in the intra-body channel increases when the frequency also increases. Moreover, the largest the distance between TX and $\mathrm{RX}$, the faster decrease of received signal power is observed. Therefore, the results from the standing model show higher slope than the ones from the sitting model. On the other hand,

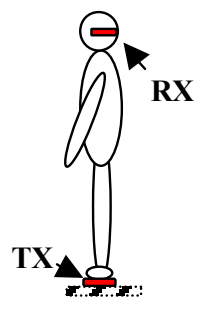

(a)

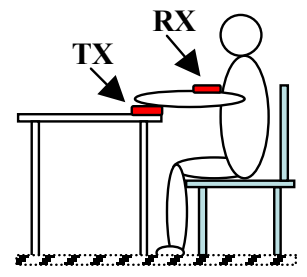

(b)
Figure 3. Measurement models: (a) standing model, (b) sitting model. 


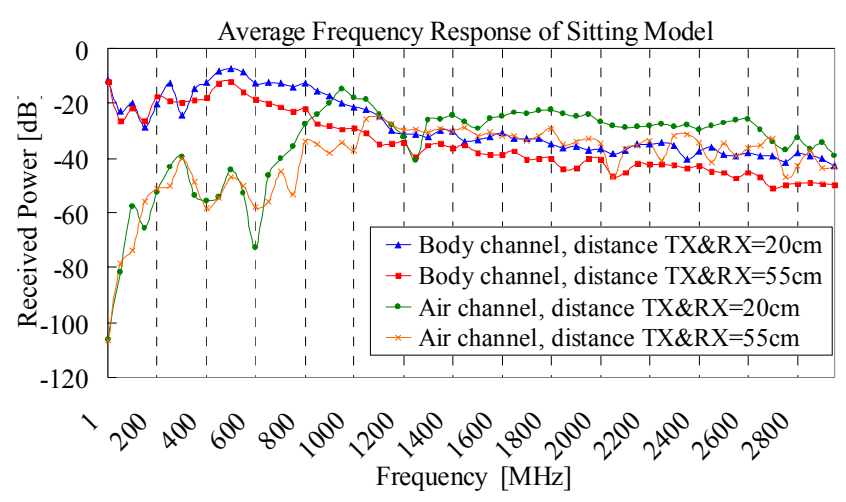

Figure 4. Frequency domain response of sitting model.



Figure 5. Frequency domain response of standing model.

the intra-body channel shows a smaller path loss than the air propagation channel as long as the radio frequency is lower than $900 \mathrm{MHz} 1050 \mathrm{MHz}$ (Fig. 4) and $800 \mathrm{MHz} \sim 850 \mathrm{MHz}$ (Fig. 5). Finally, in order to transmit data with the lowest energy consumption, we believe that the suitable frequency range for IBC is from $200 \mathrm{MHz}$ to $600 \mathrm{MHz}$, that is, the combination range of both figures with the lowest path loss.

\section{B. Time Domain Response}

For each measurement model, the average power delay profile was calculated by the inverse Fourier transform from the frequency domain data. Figs. 6 and 7 show the measurement results of the intra-body channel when the distance between TX and RX is $20 \mathrm{~cm}$ (sitting model) and $155 \mathrm{~cm}$ (ear-foot distance in standing model) respectively. The vertical axis represents the received signal power in $\mathrm{dB}$. The top of Fig. $6 \mathrm{y}$-axis represents $+20 \mathrm{~dB}$ and the scale division is $10 \mathrm{~dB}$. In case of Fig. 7, the top of $\mathrm{y}$-axis represents $-4 \mathrm{~dB}$ and the scale division is $8 \mathrm{~dB}$. The horizontal axis of both figures represents the time delay in nanoseconds from 0ns to $50 \mathrm{~ns}$ with a time division of $5 \mathrm{~ns}$. The time delay of the strongest echo of both figures is $4.125 \mathrm{~ns}$ and $12.208 \mathrm{~ns}$ respectively. The power of the strongest echo is $-13.649 \mathrm{~dB}$ (Fig. 6) and $-24.651 \mathrm{~dB}$ (Fig. 7). We observe that the time delay of the strongest echo increases and the power decreases when the distance between TX and RX is increased. Moreover, the power of the following echoes in both figures decreases gradually as the time

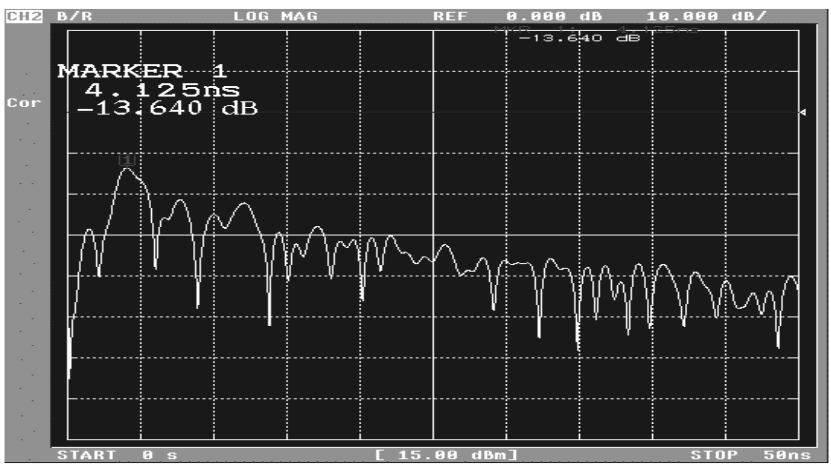

Figure 6. Intra-body propagation power delay profile when distance is $20 \mathrm{~cm}$.

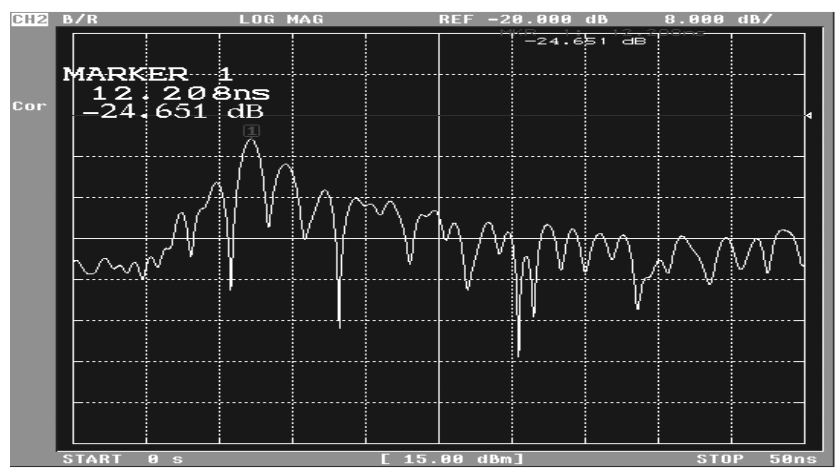

Figure 7. Intra-body propagation power delay profile when ear-foot distance.

increases. On the other hand, we observe in Fig. 6 some periodicity between the first three echoes with a time difference of $5 \mathrm{~ns}$ approximately between echoes and an attenuation of $10 \mathrm{~dB}$ between the first and second-third echoes. Finally, in case of air propagation channel, we could observe an approximately $26 \mathrm{~dB}$ power decay of the strongest echo with the same conditions as in an intra-body propagation channel.

\section{EXPERIMENT Setup For The Evaluation Of Digital MODULATION SCHEMES}

The measurements were performed based on the same environment as in the previous experiment. As it is shown in Fig. 8, we used a signal generator to generate and modulate the radio signal that was transmitted by the TX through the human body. The signal received by RX was demodulated and

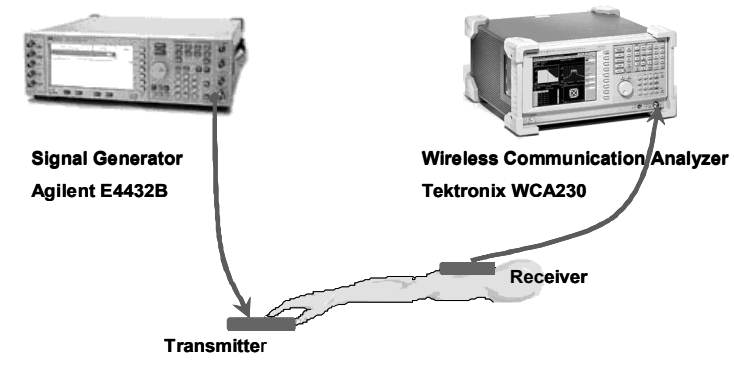

Figure 8. Experiment scenario for the evaluation of digital modulation schemes. 
TABLE II. MAIN MEASUREMENT SETUP PARAMETERS

\begin{tabular}{l|l}
\hline Parameter & Value \\
\hline Modulation scheme & MSK, BPSK, QPSK \\
\hline Carrier frequency $[\mathrm{MHz}]$ & $200,300,400,500$ \\
\hline Source data & PN9 \\
\hline Symbol rate [Ksps] & $100 \sim 2500$ \\
\hline Distance TX and RX [cm] & 20,155 \\
\hline
\end{tabular}

evaluated in a wireless communication analyzer. Table II shows the main measurement setup parameters. The frequency carriers were selected based on the most suitable frequency range determined previously. BPSK, QPSK and MSK were the three modulation schemes analyzed in the experiments as these techniques are widely used in current mobile communication systems. Several transmission rates were considered to study the maximum data rate achievable through each scheme. Short and long distance $(20 \mathrm{~cm}$ and $155 \mathrm{~cm}$, respectively) between TX and RX were set up. The present experiment employed the same TX and RX configuration used in the previous experiments.

\section{EXPERIMENT RESULTS AND DISCUSSIONS}

The constellation diagram and the error vector magnitude (EVM) are measured depending on the transmission rate and the distance between TX and RX in order to evaluate the performance of BPSK, QPSK and MSK. We show the results in case of a $200 \mathrm{MHz}$ frequency carrier. Note that similar results for the rest of frequency carriers of Table II are obtained.

In Fig. 9, firstly we investigate the constellation performance when the distance between transceivers is $20 \mathrm{~cm}$ and the symbol rate is 100Ksps (see Figs. 9a, 9b, 9c, 9d and 9e). In this setting case, we compare the performance of both intrabody and air transmission mediums. We observe that the intrabody propagation channel provides better performance with lower distortion than the air propagation channel because the body becomes a kind of wire and, therefore, the intra-body channel is not as affected by external interferences and noise sources as the air channel. Note that the figures show the comparison of both transmission mediums in case of BPSK and MSK. In case of QPSK, we also obtain similar results, that is, higher distortion with air propagation channel than with intra-body propagation channel. Regarding only the constellation results of the body transmission medium, MSK is the modulation scheme that provides the best performance because all the received symbols are within the four constellation points. BPSK and QPSK also provide good constellation diagrams with most of the received symbols close to the constellation points. On the other hand, in Figs. 9f, 9g and $9 \mathrm{~h}$, we analyze the effect of increasing the transmission rate to $2.5 \mathrm{Msps}$ and the distance between transceivers to $155 \mathrm{~cm}$ in case of intra-body transmission. The results show that, although the distortion of the received signals increases, the received symbols are still close to the constellation points in all modulation schemes.

EVM is an important metric for testing the modulation accuracy. EVM quantifies the difference between the ideal and the measured signals. Based on the recommendations from

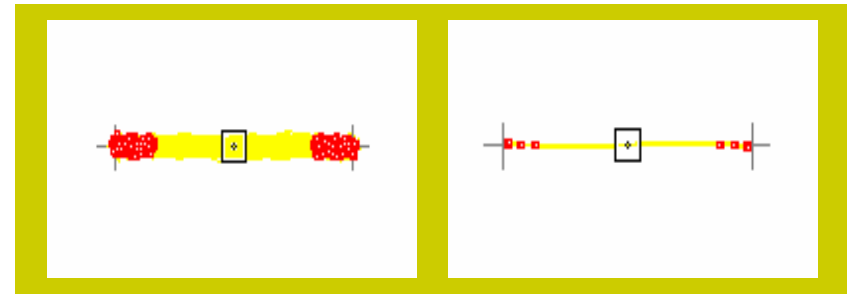

(a)

(b)

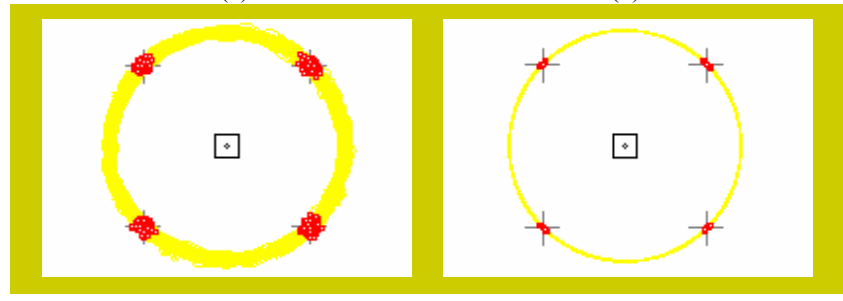

(c)

(d)

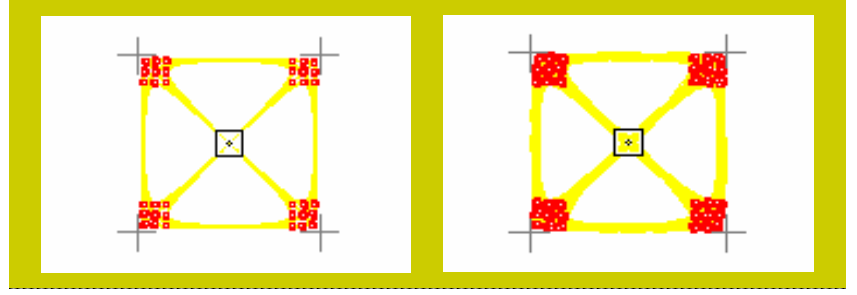

(e)

(f)

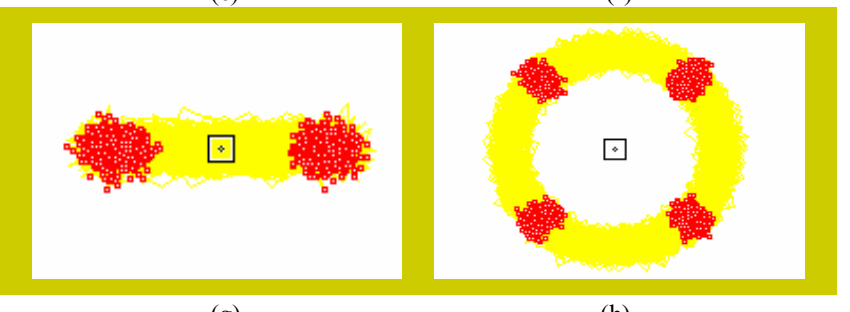

(g)

(h)

Figure 9. Constellation diagram results. (a) BPSK air channel, symbol rate $=100 \mathrm{Ksps}$ and distance $\mathrm{TX}-\mathrm{RX}=20 \mathrm{~cm}$, (b) BPSK body channel, symbol rate $=100 \mathrm{~K}$ sps and distance $\mathrm{TX}-\mathrm{RX}=20 \mathrm{~cm}$, (c) MSK air channel, symbol rate $=100 \mathrm{Ksps}$ and distance $\mathrm{TX}-\mathrm{RX}=20 \mathrm{~cm},(\mathrm{~d}) \mathrm{MSK}$ body channel, symbol rate $=100 \mathrm{Ksps}$ and distance $\mathrm{TX}-\mathrm{RX}=20 \mathrm{~cm}$, (e) QPSK body channel, symbol rate $=100 \mathrm{Ksps}$ and distance $\mathrm{TX}-\mathrm{RX}=20 \mathrm{~cm}$, (f) QPSK body channel, symbol rate $=2.5 \mathrm{Msps}$ and distance $\mathrm{TX}-\mathrm{RX}=155 \mathrm{~cm},(\mathrm{~g}) \mathrm{BPSK}$ body channel, symbol rate $=2.5 \mathrm{Msps}$ and distance $\mathrm{TX}-\mathrm{RX}=155 \mathrm{~cm}$, (h) MSK body channel, symbol rate $=2.5 \mathrm{Msps}$ and distance $\mathrm{TX}-\mathrm{RX}=155 \mathrm{~cm}$.

mobile communication standards [8]-[10], we set up that, in case of IBC, EVM shall not be worse than 7\% for MSK, $17.5 \%$ for QPSK and $20 \%$ for BPSK. Fig. 10 presents the EVM results in terms of root mean square percentage (RMS) and as a function of the symbol rate when short (i.e. $20 \mathrm{~cm}$ ) and long (i.e. $155 \mathrm{~cm}$ ) distances between TX and RX are considered. The figure shows that BPSK yields good EVM results below the threshold for both short and long distances and for all symbol rates. MSK also provides good EVM results for all symbol rates when short distance, but only for symbol rates up to 1 Msps when distance is $155 \mathrm{~cm}$. QPSK yields EVM results above EVM threshold in both $20 \mathrm{~cm}$ and $155 \mathrm{~cm}$ distance cases, meaning that QPSK is not suitable for IBC. On the other hand, we observe that MSK is the most sensible modulation scheme to the increase of transmission rate, mainly for high symbol rates. This means that when symbol rate increases the EVM 


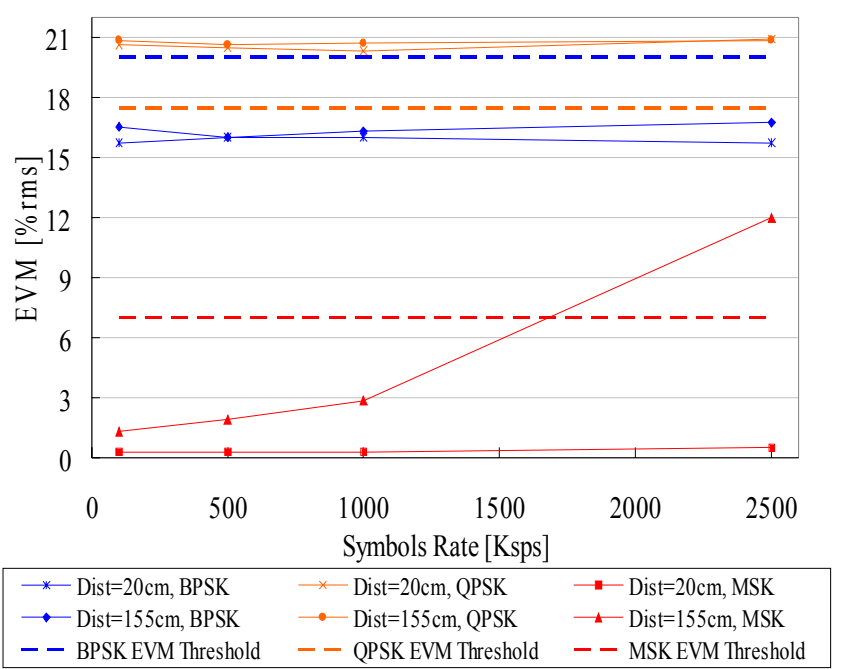

Figure 10. EVM results when distance between TX-RX is $20 \mathrm{~cm}$ and $155 \mathrm{~cm}$.

performance of MSK also increases considerably and, therefore, the modulation quality decreases.

\section{CONCLUSIONS}

In the present paper, we have investigated the transmission characteristics of the human body considering radio frequency signals up to $3 \mathrm{GHz}$. The time domain response has shown that the increase of distance between TX and RX causes an increase of the path loss and a longer time delay of the strongest echo. On the other hand, the frequency domain response has shown that the signal attenuation of the intra-body channel increases when the frequency increases. From the results, we have established a suitable frequency range for IBC between $200 \mathrm{MHz}$ to $600 \mathrm{MHz}$.

Finally, we have evaluated BPSK, QPSK and MSK modulation schemes in terms of constellation diagram and EVM. The measurements have proved that BPSK and MSK are suitable for IBC and that high transmission rates can be achieved for both short and long distances.

\section{REFERENCES}

[1] J. Agud Ruiz, S. Shimamoto, "A Study on the Transmission Characteristics of the Human Body Towards Broadband Intra-body Communications", IEEE 9th International Symposium on Consumer Electronics 2005, Macau, China, 14-16 June 2005

[2] T. G. Zimmerman, "Personal Area Networks: Near-filed Intra-Body Communication,” IBM Systems Journal, Vol .35, N. 3\&4, pp. 609-617, 1996

[3] Matsushita Electric Works Ltd., 2004, www.mew.co.jp/epress/2004/0409-02.htm

[4] M. Shinagawa et al., "A Near-Field-Sensing Transceiver for Intrabody Communication Based on the Electrooptic Effect," IEEE Transactions on instrumentation and measurement, Vol. 53, NO. 6, december 2004

[5] K. Hachisuka Post et al., "Development and Performance Analysis of an Intra-Body Communication Device," Proc. of 12th International Conference on Solid State Sensors, Actuators and Microsystems, Boston, pp. 1722-1725, June 2003

[6] K.Fujii, K. Ito, "Evaluation of the Received Signal Level in Relation to the Size and Carrier Frequencies of the Wearable Device Using Human
Body as a Transmission Channel," Proc. of 2004 Antennas and Propagation Society Symposium, pp. 105-108, June 2004

[7] World Health Organization, "Electromagnetic Fields $(300 \mathrm{~Hz}$ to 300GHz)," 1993, www.inchem.org/documents/ehc/ehc/ehc137.htm

[8] ETSI GSM 5.05 Standard v.8.4.1: "Digital Cellular Telecommunications System (phase 2+), Radio Transmission and Reception”, 1999

[9] 3GPP TS 25.101 v.6.8.0: "User Equipment (UE) radio transmission and reception (FDD)", June 2005

[10] K. T. Le, "Designing a ZigBee-ready IEEE 802.15.4-compliant radio transceiver", www.rfdesign.com, November 2004 